\title{
Qualitative Modeling for Magnetization Curve
}

\author{
Pei-Hwa Huang \\ Associate Professor, Department of Electrical Engineering, National Taiwan Ocean University, Keelung, Taiwan. \\ Yu-Shuo Chang \\ Graduate Student, Department of Electrical Engineering, National Taiwan Ocean University, Keelung, Taiwan.
}

Follow this and additional works at: https://jmstt.ntou.edu.tw/journal

Part of the Electrical and Computer Engineering Commons

\section{Recommended Citation}

Huang, Pei-Hwa and Chang, Yu-Shuo (2000) "Qualitative Modeling for Magnetization Curve," Journal of Marine Science and Technology. Vol. 8: Iss. 2, Article 1.

DOI: $10.51400 / 2709-6998.2455$

Available at: https://jmstt.ntou.edu.tw/journal/vol8/iss2/1

This Research Article is brought to you for free and open access by Journal of Marine Science and Technology. It has been accepted for inclusion in Journal of Marine Science and Technology by an authorized editor of Journal of Marine Science and Technology. 


\title{
QUALITATIVE MODELING FOR MAGNETIZATION CURVE
}

\author{
Pei-Hwa Huang* and Yu-Shuo Chang**
}

Keywords: Magnetization curve, Qualitative modeling, Fuzzy modeling.

\begin{abstract}
This paper studies the derivation of a qualitative description for the magnetization curve of saturable magnetic materials. From a set of numerical input-output data, a fuzzy model, which is a collection of fuzzy IF-THEN rules for describing the studied system, is first constructed for the magnetization curve. A procedure consisting of a fuzzy clustering method and a rule processing algorithm is then employed for processing the constructed fuzzy modeling rules to extract the qualitative properties of the curve. The proposed approach is objective in that it can yield the system qualitative features without domain experts' knowledge. An example is demonstrated to illustrate the qualitative modeling procedure of the magnetization curve.
\end{abstract}

\section{INTRODUCTION}

The ferromagnetic material plays an important role in the energy conversion process of electric power engineering [1]. Analysis of devices made of ferromagnetic materials requires a knowledge of the physical properties of the material used, among which the magnetization curve is the most fundamental one and is characteristic of nonlinearity due to saturation. Numerous mathematical modeling methods [2-15] have been devised for the representation of magnetization curves. However, the issue of deriving a qualitative model for describing linguistically the nature of magnetization curves has not been addressed.

Qualitative modeling and reasoning have been important issues in the area of artificial intelligence since 1970's [16-21]. On the other hand, the fuzzy model, which is a collection of fuzzy IF-THEN rules for describing the studied system, has been recognized as a powerful tool for modeling and control of dynamic

Paper Received Jul. 28, 2000; Accepted Sep. 8, 2000. Author for Correspondence: Pei-Hwa Huang.

*Associate Professor, Department of Electrical Engineering, National Taiwan Ocean University, Keelung, Taiwan.

**Graduate Student, Department of Electrical Engineering, National Taiwan Ocean University, Keelung, Taiwan. systems [22-25]. Recently some studies [26-31] have been reported concerning the application of fuzzy models to the study of qualitative modeling. A fuzzymodeling-based qualitative modeling approach $[30,31]$ will be employed for this research.

In this paper, we aim to study the modeling of magnetization curves in a qualitative perspective. The point is to suggest a systematic procedure for deriving a qualitative description for the magnetization curve. In practice the data of a magnetization curve is obtained by measuring the magnetic flux density $B$ (output quantity) corresponding to a given magnetic field intensity $H$ (input quantity). A fuzzy model is first constructed to approximate the given data quantitatively. Then a qualitative modeling approach $[30,31]$ is to be presented for further processing of the identified fuzzy modeling rules to obtain a qualitative model for the magnetization curve. The approach consists of two parts: a fuzzy clustering method to define fuzzy sets on the input and output spaces along with a rule processing algorithm for summarizing the constructed fuzzy modeling rules to derive a set of qualitatively descriptive rules for describing the given input-output data. The proposed approach is objective in that it can reveal the system qualitative features without the need for domain experts' knowledge. A sample example will be shown to illustrate the proposed procedure.

\section{METHODOLOGY}

Based on the concept of fuzzy modeling, a qualitative modeling $[30,31]$ approach will be discussed in this section. Fuzzy modeling is adopted for giving an mathematical approximation of the magnetization curve data. Then a procedure will be presented for processing the fuzzy modeling rules to arrive at a qualitative model.

\section{Fuzzy Modeling}

In this study, we consider the single-input-singleoutput fuzzy model, composed by $m$ fuzzy rules $R^{i}, i=$ $1,2, \ldots, m$, which is of the form 


$$
R^{i} \text { : IF } x \text { is } A_{i} \text { THEN } y \text { is } D_{i}
$$

where $x$ and $y$ are the input and output variables, respectively, and both $A_{i}$ and $D_{i}$ are fuzzy sets. The fuzzy sets $A_{i}$ 's are assumed to have Gaussian-type membership functions focused at $x_{i}^{*}$ :

$$
A_{i}(x)=\exp \left(\frac{-\left(x-x_{i}^{*}\right)^{2}}{2 \sigma_{i}^{2}}\right)
$$

and $D_{i}$ 's are assumed to be defined by their respective centroids $y_{i}^{*}$ s. The fuzzy rule expressed in (1) can be interpreted linguistically as

$$
R^{i} \text { : IF } x \text { is close to } x_{i}^{*} \text { THEN } y \text { is close to } y_{i}^{*} \text {. }
$$

From the system numerical input-output data, the fuzzy model in the form of (1) can be constructed by using the mountain method [32, 33] for structure identification and the back-propagation method [34] for parameter identification. The identified fuzzy model, i.e. the set of fuzzy rules representating the system behavior, is then to be processed by the procedure described in the following subsection to acquire a qualitative model for the system under study.

\section{Qualitative Modeling}

Here we present a procedure for deriving a qualitative model based on a fuzzy model which is composed of a set of fuzzy rules in the form of (1). The main idea is to reduce the number of modeling rules and to extract the system qualitative nature simultaneously. The procedure comprises two main parts, namely a fuzzy clustering method and a rule processing algorithm.

\subsection{Fuzzy clustering}

Based on the fuzzy c-means method $[35,36]$, the below-described fuzzy clustering method is presented for defining fuzzy sets and linguistic labels on the input space and the output space. These fuzzy sets and linguistic labels form the basis for the processing of identified fuzzy modeling rules. Denote the given set of system input-output data as $\left\{\left(x_{i}, y_{i}\right)\right\}$. The proposed fuzzy clustering method is summarized as:

1. Specify the required number of fuzzy clusters, i.e. the number of fuzzy sets (linguistic labels), for the input space $x$ and the output space $y$. Then perform fuzzy clustering on the input data $\left\{x_{i}\right\}$ and the output data $\left\{y_{i}\right\}$ using the fuzzy c-means method to obtained the desired fuzzy sets. The fuzzy sets thus formed have discrete membership grades, which can be depicted as the point line in Fig. 1(a).
2. Let $\mu_{i k}$ be the membership value of the $k$ th data point in the $i$ th fuzzy set. Approximate all the membership values using the following criterion:

$$
\begin{aligned}
& \mu_{i k} \leftarrow 1.0, \text { if } \mu_{i k} \geq 0.95, \\
& \mu_{i k} \leftarrow 0.0, \text { if } \mu_{i k} \leq 0.05, \\
& \mu_{i k} \leftarrow \mu_{i k}, \quad \text { otherwise. }
\end{aligned}
$$

After the above approximation, we can obtain for each fuzzy set the parameters $b$ and $c$ which are the maximum and minimum data respectively achieving the membership value of 1.0, as shown in Fig. 1(a).

3. For each fuzzy set, perform linear regression analysis on those data with approximated membership values less than $b$ and those data having approximated membership grades greater than $c$ to get the parameters $a$ and $d$ shwon in Fig. 1(b).

4. Finally the discrete membership grade distribution of each fuzzy set is approximated by a trapezoidal membership function with parameters $(a, b, c, d)$ as shown in Fig. 1. We can then denote each fuzzy set by an appropriate linguistic label, e.g. NL(Negative Large).

In summary, we aim to define on the input and output spaces some linguistic labels stemming from fuzzy sets equipped with trapezoidal membership functions. These linguistic labels will be the basis for transforming a set of fuzzy modeling rules into the system qualitative description.

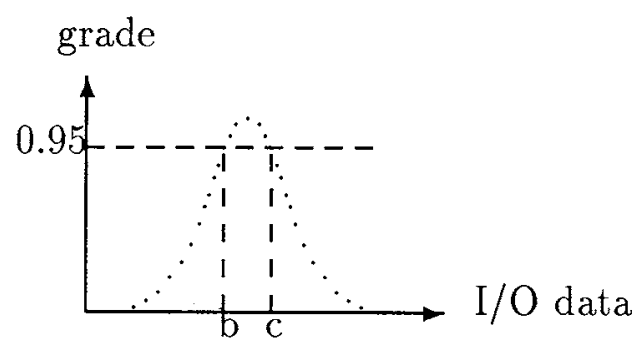

(a)

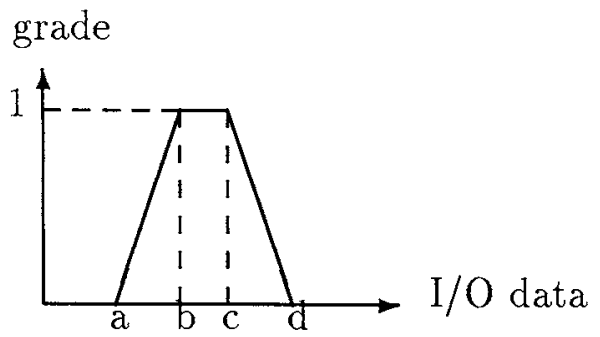

(b)

Fig. 1. (a) Membership grade values by fuzzy c-means method. (b) Approximated trapezoidal membership function. 


\subsection{Rules processing}

After defining the fuzzy sets (linguistic labels) on the input space and the output space, the set of identified fuzzy model rules is to be processed by the following steps to derive the qualitative model. The key point is to reduce the number of and to summarize the fuzzy rules by first converting them into linguistic rules and then combining the linguistic rules.

Step 1 The fuzzy rules are sorted in the ascending order of $x_{i}^{*}$ s values. This makes the fuzzy model an orderly description of the system and will make the processing of modeling rules more efficient.

Step 2 For each fuzzy rule $R^{i}$, find the respective lingusitic labels in which $x_{i}^{*}$ and $y_{i}^{*}$ attains their maximum membership values. Substitute the linguistic labels for the corresponding $A_{i}$ and $B_{i}$. In this way, we arrive at a set of linguistic rules from the original fuzzy rules. That is, we give a linguistic interpretation for each fuzzy modeling rule.

Step 3 Combine those adjacent linguistic rules of which both the premises and the consequents are the same. That is, we use a single rule to represent a group of linguistically identical rules. This will achieve a reduction in the number of rules.

Step 4 For those adjacent linguistic rules having the same label in the premise, express them by a single rule which has the same premise and of which the consequent gives a description of the system dynamic behavior by integrating the original consequents. In this way, we obtain only one model rule for each linguistic label of the input space.

By using the above four steps, we finally arrive at a qualitative model, a set of linguistic rules describing the system dynamic behaviors qualitatively. It is worth emphasizing that for a single-input-single-output system, the number of modeling rules should be equal to the number of linguistic labels in the input space.

\section{EXAMPLE}

In this section, the proposed approach will be applied to the qualitative modeling study of a sample input-output data set of a magnetization curve. The data set is taken from a magnetization curve plot in [1, p. 18] and there are forty data points in the magnetic flux density-magnetic field intensity $(B-H)$ plane and is shown as Fig. 2 in which both the regular and the semi-logarithmic plots are given.

As mentioned in the previous section, we are to identify a fuzzy model for the given data. According to
(1) and (3), the $i$-th fuzzy rule for describing the magnetization curve is of the following form

$$
R^{i} \text { : If } H \text { is } A_{i} \text {, THEN } B \text { is } D_{i} \text {, }
$$

where $H$ and $B$ denote magnetic field intensity (the input variable) and magnetic flux density (the output variable), respectively. Assume that $A_{i}$ has a Gaussian-type membership function focused at $x_{i}^{*}$, as in (2), and $D_{i}$ is defined by its centroid $y_{i}^{*}$, then the rule in (4) can be interpreted as

$$
R^{i} \text { : IF } H \text { is close to } x_{i}^{*} \text { THEN } B \text { is close to } y_{i}^{*} \text {. }
$$

The fuzzy modeling rules for the magnetization curve can be identified and are summarized as Table 1. Note that there are totally twenty four rules with a mean square modeling error $3.7966 \times 10^{-5}$.

After figuring out the fuzzy model, we are going to process and summarize these rules for acquiring qualitative information. First of all, cluster analysis is performed on the original data set using the modified fuzzy c-means clustering method. The number of fuzzy clusters is set to be three for each of the input and output domains. The resultant fuzzy partitions for the input and output spaces are shown in Figure 3.

Fuzzy rules listed in Table 1 are to be processed by the proposed rule processing algorithm. The following steps and Tables 1-4 summarize the procedure of rule processing.

Step 1 The fuzzy rules in Table 1 have been sorted in the ascending order of $x_{i}^{*}$ s values.

Step 2 With the fuzzy partitions shown in Fig. 3 the fuzzy model rules listed in Table 1 can be trans-
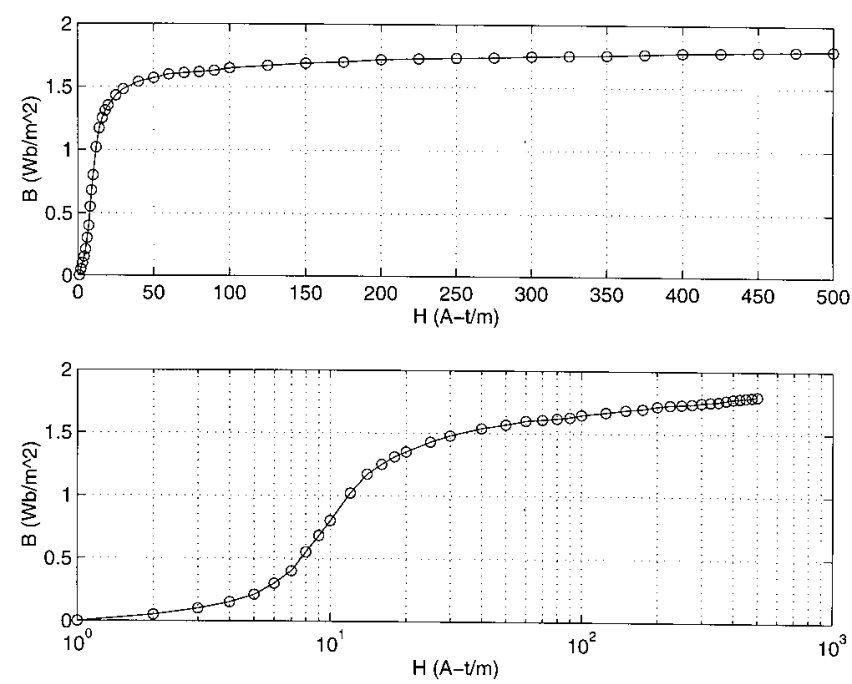

Fig. 2. Magnetization curve. 
Table 1. Fuzzy model rules. The rules have been sorted in the ascending order of $x_{i}^{*}$ value, as in Step 1.

\begin{tabular}{ccc}
\hline Rule & $x_{i}^{*}$ & $y_{i}^{*}$ \\
\hline$R^{1}$ & 5.29 & -0.082 \\
$R^{2}$ & 10.99 & 1.130 \\
$R^{3}$ & 19.07 & 1.362 \\
$R^{4}$ & 29.65 & 1.482 \\
$R^{5}$ & 40.00 & 1.540 \\
$R^{6}$ & 60.00 & 1.600 \\
$R^{7}$ & 80.00 & 1.620 \\
$R^{8}$ & 90.00 & 1.643 \\
$R^{9}$ & 125.00 & 1.670 \\
$R^{10}$ & 150.00 & 1.690 \\
$R^{11}$ & 175.00 & 1.700 \\
$R^{12}$ & 200.00 & 1.720 \\
$R^{13}$ & 225.00 & 1.730 \\
$R^{14}$ & 250.00 & 1.735 \\
$R^{15}$ & 275.00 & 1.740 \\
$R^{16}$ & 300.00 & 1.750 \\
$R^{17}$ & 325.00 & 1.755 \\
$R^{18}$ & 350.00 & 1.760 \\
$R^{19}$ & 375.00 & 1.770 \\
$R^{20}$ & 400.00 & 1.780 \\
$R^{21}$ & 425.00 & 1.785 \\
$R^{22}$ & 450.00 & 1.790 \\
$R^{23}$ & 475.00 & 1.795 \\
$R^{24}$ & 500.00 & 1.800 \\
\hline
\end{tabular}

formed into linguistic rules listed in Table 2.

Step 3 The number of rules of the linguistic rules listed in Table 2 can be reduced by combining rules having the same linguistic descriptions and the resultant new set of linguistic rules are tabulated as Table 3.

Step 4 Based on Table 3, we are finally in a position to derive a qualitative model for the system under study by integrating rules having the same premise. Table 4 shows the final three qualitative rules for the system.

According to Table 4 we have the following threerule qualitative model for the magnetization curve shown in Fig. 2.

$Q^{1}$ : If $H$ is SMALL THEN $B$ is increased from SMALL to MEDIUM and then to LARGE.

$Q^{2}$ : If $H$ is MEDIUM THEN $B$ is LARGE.

$Q^{3}$ : IF $H$ is LARGE THEN $B$ is LARGE.

Note that since the input space is partitioned into three fuzzy regions, there will be three qualitative descriptive rules, i.e. $Q^{1}, Q^{2}$ and $Q^{3}$, one for each fuzzy
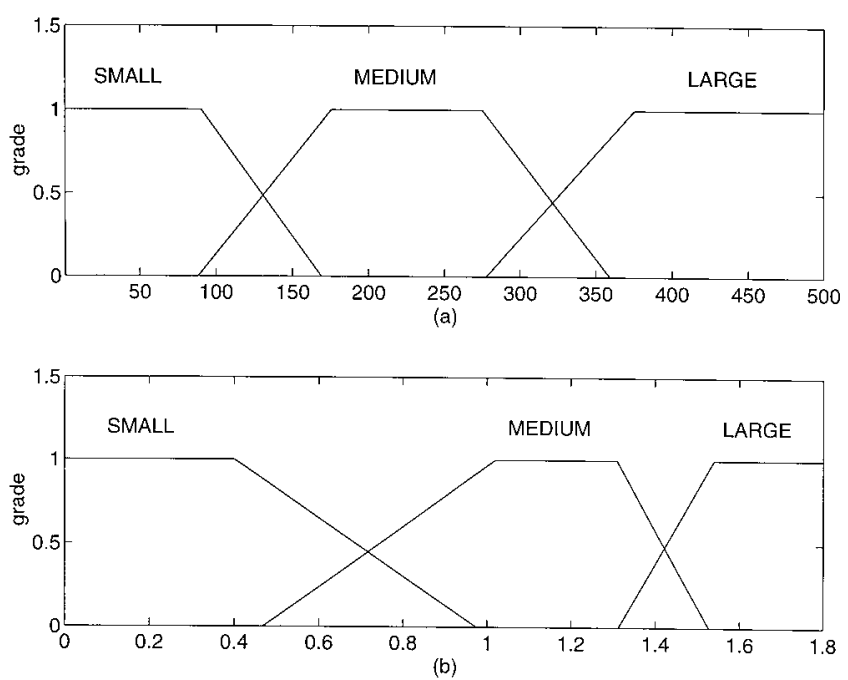

Fig. 3. Fuzzy partitions for input and output spaces. Both the input and output spaces are partitioned into S(Small), M(Medium), and L (Large).

Table 2. Linguistic rules obtained by Step 2.

\begin{tabular}{lll}
\hline Rule & $X$ & $Y$ \\
\hline$R^{1}$ & $\mathrm{~S}$ & $\mathrm{~S}$ \\
$R^{2}$ & $\mathrm{~S}$ & $\mathrm{M}$ \\
$R^{3}$ & $\mathrm{~S}$ & $\mathrm{M}$ \\
$R^{4}$ & $\mathrm{~S}$ & $\mathrm{~L}$ \\
$R^{5}$ & $\mathrm{~S}$ & $\mathrm{~L}$ \\
$R^{6}$ & $\mathrm{~S}$ & $\mathrm{~L}$ \\
$R^{7}$ & $\mathrm{~S}$ & $\mathrm{~L}$ \\
$R^{8}$ & $\mathrm{~S}$ & $\mathrm{~L}$ \\
$R^{9}$ & $\mathrm{~S}$ & $\mathrm{~L}$ \\
$R^{10}$ & $\mathrm{M}$ & $\mathrm{L}$ \\
$R^{11}$ & $\mathrm{M}$ & $\mathrm{L}$ \\
$R^{12}$ & $\mathrm{M}$ & $\mathrm{L}$ \\
$R^{13}$ & $\mathrm{M}$ & $\mathrm{L}$ \\
$R^{14}$ & $\mathrm{M}$ & $\mathrm{L}$ \\
$R^{15}$ & $\mathrm{M}$ & $\mathrm{L}$ \\
$R^{16}$ & $\mathrm{M}$ & $\mathrm{L}$ \\
$R^{17}$ & $\mathrm{~L}$ & $\mathrm{~L}$ \\
$R^{18}$ & $\mathrm{~L}$ & $\mathrm{~L}$ \\
$R^{19}$ & $\mathrm{~L}$ & $\mathrm{~L}$ \\
$R^{20}$ & $\mathrm{~L}$ & $\mathrm{~L}$ \\
$R^{21}$ & $\mathrm{~L}$ & $\mathrm{~L}$ \\
$R^{22}$ & $\mathrm{~L}$ & $\mathrm{~L}$ \\
$R^{23}$ & $\mathrm{~L}$ & $\mathrm{~L}$ \\
$R^{24}$ & $\mathrm{~L}$ & $\mathrm{~L}$ \\
\hline
\end{tabular}

region. Investigation of the final qualitative model and Fig. 2 reveals that the acquired model $\left\{Q^{1}, Q^{2}, Q^{3}\right\}$ does provide a qualitative description for the saturation phenomenon of the magnetization curve. 
Table 3. Linguistic rules obtained by Step 3.

\begin{tabular}{cccc}
\hline Rule & $\mathrm{X}$ & $\mathrm{Y}$ & Rules combined \\
\hline$R^{1}$ & $\mathrm{~S}$ & $\mathrm{~S}$ & $R^{1}$ \\
$S^{1}$ & $\mathrm{~S}$ & $\mathrm{M}$ & $R^{2}, R^{3}$ \\
$S^{2}$ & $\mathrm{~S}$ & $\mathrm{~L}$ & $R^{4}, \ldots, R^{9}$ \\
$S^{3}$ & $\mathrm{M}$ & $\mathrm{L}$ & $R^{10}, \ldots, R^{16}$ \\
$S^{4}$ & $\mathrm{~L}$ & $\mathrm{~L}$ & $R^{17}, \ldots, R^{24}$ \\
\hline
\end{tabular}

Table 4. Qualitative rules obtained by Step 4.

\begin{tabular}{cccc}
\hline Rule & $\mathrm{X}$ & $\mathrm{Y}$ & Rules combined \\
\hline$Q^{1}$ & $\mathrm{~S}$ & $\mathrm{~S} \rightarrow \mathrm{M} \rightarrow \mathrm{L}$ & $R^{1}, S^{1}, S^{2}$ \\
$Q^{2}$ & $\mathrm{M}$ & $\mathrm{L}$ & $S^{3}$ \\
$Q^{3}$ & $\mathrm{~L}$ & $\mathrm{~L}$ & $S^{4}$ \\
\hline
\end{tabular}

\section{CONCLUSION}

In this paper, we have proposed an approach for the qualitative modeling of magnetization curves. Given the numerical input-output data, a set of fuzzy modeling rules is identified for approximating the magnetization curve. Then the qualitative model is derived by defining fuzzy sets and linguistic labels on the input and output spaces and by processing the fuzzy rules of the identified fuzzy model. The proposed modeling approach has been tested and validated by a sample example.

\section{REFERENCES}

1. A. E. Fitzgerald, C. Kingsley, Jr., and S. D. Umans, Electric Machinery, Fifth edition, McGraw-Hill (1990).

2. M. K. El-Sherbiny, "Representation of the magnetization characteristic by a sum of exponentials," IEEE Trans. on Magnetics, Vol. 9, pp. 60-61 (1973).

3. W. K. Macfadyen, R. R. Simpson, R. D. Slater, and W. S. Wood, "Representation of magnetisation curves, including hysteresis, by exponential series," IEE Proceedings, Vol. 120, 902-904 (1973).

4. J. H. Hwang and W. Lord, "Exponential series for B/H curve modeling," IEE Proceedings, Vol. 123, pp. 559560 (1976).

5. S. Prusty and M. V. S. Rao, "A direct piecewise linearized approach to convert rms saturation characteristic to instantaneous saturation curve," IEEE Trans. on Magnetics, Vol. 16, pp. 156-160 (1980).

6. J. Rivas, J. M. Zamarro, E. Martin, and C. Pereira, "Simple approximation for magnetization curves and hysteresis loops," IEEE Trans. on Magnetics, Vol. 17, pp. 1498-1502 (1981).
7. B. Forghani, E. M. Freeman, D. A. Lowther, and P. P. Silvester, "Interactive modelling of magnetisation curves," IEEE Trans. on Magnetics, Vol. 18, pp. 10701072 (1982).

8. S. Prusty and M. V. S. Rao, "A novel approach for predetermination of magnetization characteristics of transformers including hysteresis," IEEE Trans. on Magnetics, Vol. 20, 607-612 (1984).

9. M. A. Mahmoud and R. W. Whitehead, "Piecewise fitting function for magnetisation characteristics," IEEE Trans. on Power Systems and Apparatus, Vol. 104, 1822-1824 (1985).

10. D. A. Lowther and P. P. Silvester, Computer-Aided Design in Magnetics, Springer-Verlag (1986).

11. D. C. Jiles and D. L. Atherton, "Theory of ferromagnetic hysteresis," Journal of Magnetism and Magnetic Materials, Vol. 61, pp. 48-60 (1986).

12. J. D. Greene and C. A. Gross, "Nonlinear modeling of transformers," IEEE Trans. on Power Systems and Apparatus, Vol. 24, 434-438 (1988).

13. V. Ostović, Dynamics of Saturated Electric Machines, Springer-Verlag (1989).

14. E. Santini and M. Vinci, "Fast and efficient techniques for interpolation of B-H curves," in P. P. Silvester (ed.), Software Applications in Electrical Engineering, Computational Mechanics Publications (1993).

15. D. Dolinar, J. Pihler, and B. Grčar, "Dynamic model of a three-phase power transformer," IEEE Trans. on Power Delivery, Vol. 8, pp. 1811-1819 (1993).

16. J. de Kleer and J. S. Brown, "A qualitative physics based on confluences," Artificial Intelligence, Vol. 24, pp. 783 (1984).

17. K. D. Forbus "Qualitative process theory," Artificial Intelligence, Vol. 24, pp. 85-168 (1984).

18. B. Kuipers, "Qualitative simulation," Artificial Intelligence, Vol. 29, pp. 289-338 (1986)

19. D. S. Weld and J. de Kleer (eds.), Readings in Qualitative Reasoning about Physical Systems, Morgan Kaufmann (1990).

20. B. Kuipers, Qualitative Reasoning: Modeling and Simulation with Incomplete Knowledge, MIT Press (1994).

21. H. Werthner, Qualitative Reasoning: Modeling and the Generation of Behavior, Springer-Verlag (1994).

22. L. A. Zadeh, "Fuzzy sets," Information and Control, Vol. 8, pp. 338-353 (1965).

23. T. Takagi and M. Sugeno, "Fuzzy identification of system and its applications to modeling and control," IEEE Trans. on Systems, Man, and Cybernetics, Vol. 15, pp. 116-132 (1985).

24. B. J. Bezdek, "Fuzzy models-What are they, and why?" IEEE Trans. on Fuzzy Systems, Vol. 1, pp. 1-6 (1993).

25. R. R. Yager and D. P. Filev, Essentials of Fuzzy Modeling and Control, John Wiley \& Sons (1994). 
26. L. A. Zadeh, "Outline of a new approach to the analysis of complex systems and decision processes," IEEE Trans. on Systems, Man, and Cybernetics, Vol. 3, pp. 28-44 (1973).

27. B. D'Ambrosio, Qualitative Process Theory Using Linguistic Variables, Springer-Verlag (1989).

28. M. Sugeno and T. Yasukawa, "A fuzzy-logic-based approach to qualitative modeling," IEEE Trans. on Fuzzy Systems, Vol. 1, pp. 7-31 (1993).

29. Q. Shen and R. Leitch, "Fuzzy qualitative simulation," IEEE Trans. on Systems, Man, and Cybernetics, Vol. 23, pp. 1038-1061 (1993).

30. Yu-Shuo Chang, Qualitative Description of Nonlinear Systems via Fuzzy Modeling, M. S. Thesis (in Chinese), National Taiwan Ocean University (1995).

31. P. H. Huang and Y. S. Chang, "Fuzzy rules based qualitative modeling," Proc. of the Fifth IEEE International Conference on Fuzzy Systems, pp. 1261-1265 (1996).

32. R. R. Yager and D. P. Filev, "Learning of fuzzy rules by mountain clustering," Proc. of the SPIE - the International Society for Optical Engineering, Vol. 2061, pp. 246-254 (1993).

33. R. R. Yager and D. P. Filev, "Approximate clustering via the mountain method," IEEE Trans. on Systems, Man, and Cybernetics, Vol. 24, pp. 1279-1284 (1994).

34. L. X. Wang and J. M. Mendel, "Back-propagation fuzzy system as nonlinear dynamic system identifiers," Proceedings of the First IEEE International Conference on Fuzzy Systems, pp. 1409-1416 (1992).

35. J. C. Bezdek, Pattern Recognition with Fuzzy Objective Function Algorithm, Plenum Press (1981).

36. J. C. Bezdek and S. K. Pal, Fuzzy Models for Pattern Recognition, IEEE Press (1992).

\section{磁化曲線之定性模型}

$$
\text { 黄培華張育碩 }
$$

國立台灣海洋大學電機工程學系

$$
\text { 摘 要 }
$$

本文針對飽和磁性材料, 推導其磁化曲線的定 性描述。針對輸入輸出數據資料, 首先建立由模糊 $\lceil$ 若…, 則…」法則所組成的模糊模型, 來描述所研 究的磁化曲線。接下來, 利用一套包括模糊聚類以及 法則處理的演算程序, 將已建立的模糊模型法則加以 處理, 以提取出磁化曲線的定性性質。本文提出的方 法不需専家知識即可導出研究對象的定性特徵, 因此 具有客觀性。文中以一實例説明磁化曲線定性模型的 推導過程。

關鍵詞：磁化曲線、定性模型、模糊模型。 